\title{
Comparative Study of the Biomechanical Factors in Range of Motion, Muscle Activity, and Vertical Ground Reaction Force between a Forward Lunge and Backward Lunge
}

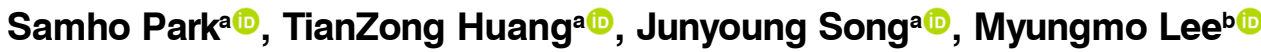 \\ ${ }^{a}$ Department of Physical Therapy, Graduate School, Daejeon University, Republic of Korea

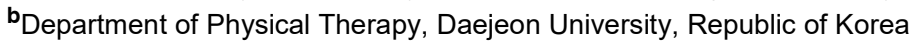

Objective: The purpose of this study was to examined the kinematic relationship and differences through the range of motion (ROM), muscle activity, and vertical ground reaction force (VGRF) during forward and backward lunge movements, which are effective in improving muscle strength and balance ability of the lower extremities, and to provide clinical information on more efficient lunge movements.

\section{Design: Cross-sectional study}

Methods: Fifteen adult males who met the selection criteria were tested for their dominant feet.Forward and backward lunges were then performed, and the ROM, muscle activity, and VGRF were measured for kinematic analysis during the lunge movement.The differences betweenthe forward lunge and backward lunge intervention were examined using a paired t-test.

Results: A significant increase in the ROM of the knee and ankle was observed during the forward and backward lunges ( $\mathrm{p}<0.05$ ). In addition, in terms of the muscle activity, the peak values of the vastus medialis oblique (VMO) and VGRF also showed a significant increase in the forward lunge compared to the backward lunge $(p<0.05)$.

Conclusions: This study showed an increase in VGRF peak value, knee and ankle ROM, and VMO muscle activity during forward lunge. Based on these results, it is considered necessary to apply differently depending on the direction of progress in consideration of the musculoskeletal situation and physical ability during the lunge movement.

Key Words: Electromyography, Exercise, Kinematics, Quadriceps Muscle, Weight-Bearing

\section{Introduction}

The patella is located between the femur and tibia, and the knee joint acts as a pulley [1]. In general, the area of contact between the patella and the femur is larger and thicker than the medial articular surface, resulting in a more significant load [2]. Abnormal alignment of the patella and an imbalance in muscle strength are pathological causes of patellofemoral pain syndrome (PFPS) [3]. The imbalance of muscle strength causes chronic pain by repeating functionally incorrect movements and decreasing motor performance [4]. For the correct alignment of the patella, strength exercises are essential for ensuring the balance and stability of the medial and lateral masses [5].

Strength training can be divided into a closed-kinetic chain (CKC) exercise that is performed while the distal limb is fixed and an open-kinetic chain (OKC) exercise that is performed in the free state without a distal limb [6]. Among these exercises, CKC exercise mobilizes many muscles of the body segment. Moreover, as the distal limb is fixed, it provides stability through a joint compression force and promotes a proprioceptive sensation[7]. CKC exercise is essential for rehabilitation

Received: Apr 8, 2021 Revised: May 12, 2021 Accepted: May 14, 2021

Corresponding author: Myungmo Lee(ORCID https://orcid.org/0000-0002-2192-1701)

Department of Physical Therapy, Daejeon University

62, Daehak-ro, Dong-gu, Daejeon city, Republic of Korea [34520]

Tel: + 82-10-9190-1770 Fax: 82-42-280-4295, E-mail: mmlee@dju.kr

This is an Open-Access article distributed under the terms of the Creative Commons Attribution Non-Commercial License (http://creativecommons.org/licenses/ by-nc/4.0) which permits unrestricted non-commercial use, distribution, and reproduction in any medium, provided the original work is properly cited.

Copyright @ 2021 Korean Academy of Physical Therapy Rehabilitation Science 
exercise for static and dynamic stabilization of the joints [8]. Proper exercise prescription improves the function of the musculoskeletal system significantly [9].

Typical CKC exercises include squat, lunge, and step-up. These exercises can train multiple muscles simultaneously without any equipment through multi-joint, weight bearing through the lower extremities [10]. The lunge is a difficult exercise among CKC exercises because it requires physical abilities, such as physical strength, agility, and muscular endurance [11]. This exercise effectively increases the strength of the hip, knee, and ankle joints and increases the eccentric contraction of the quadriceps muscle. In particular, it is an effective exercise to maintain the balance of the left and right muscle strength around the knee joint by increasing the ratio of the muscle activity of the vastus medialis oblique (VMO) to vastus lateralis (VL) [12].

The lunge moves the other foot forward, backward, or sideward while one foot supports the weight. Moreover, the demand for a sense of balance is added compared to the squat because it performs a movement [13]. In addition, due to the movement of the center of gravity (COG) on the ground, the lunge regulates the external force applied to the body between movements, and many muscles are mobilized to maintain stability [14]. Therefore, the types of lunges selected for exercise design for various purposes are classified into the forward lunge, backward lunge, and side lunge according to the direction of the lower limb to be moved [13]. The forward lunge and the backward lunge have a common point in which movement occurs around the mediolateral axis inthe sagittal plane. It is a bilateral movement in which an external force from COG movement is applied to both lower limbs [15].

Several studies have shown that lunge exercise is effective in improving the muscle strength and balance ability of the lower extremities [16]. On the other hand, research on biomechanical analysis according to the forward and backward lunges is insufficient[13]. Accordingly, the purpose of this study is to investigate the kinematic relationship and difference through range of motion (ROM), muscle activity, and vertical ground reaction force (VGRF) during forward and backward lunge movements, and to provide clinical information on efficient lunge movements.

\section{Methods}

\section{Participants}

The participants were 22 healthy males in their twenties recruited through a recruitment questionnaire distributed in advance to D University in Daejeon City from September 1to September 30, 2020. The participants understood the purpose and contents of the study and voluntarily participated in the intervention. The inclusion criteria were those who could perform lunge movements, had no abnormalities in lower extremity muscle strength and gait, could understand the purpose of the study, and agreed to participate. Those with the following were excluded: open wounds at the electrode attachment site, a history of surgery on both lower extremities, musculoskeletal disorders in both lower extremities, and Cumberland ankle instability tool (CAIT) with 24 points or less [18]. Of the 22 participants recruited, the final 15 people who met the selection criteria participated in the experiment. All participants confirmed their participation in the study according to the Helsinki Declaration.

\section{Procedures}

This study was a cross-sectional study design. G-power 3.19 software was used to calculate the sample size. The alpha level and power were set to 0.05 and 0.8 , respectively. According to previous studies, the effective size was set to 0.75 , meaning that at least 13 participants were required [19]. The minimum number of participants to be selected was 15 ,considering a dropout rate of $10 \%$. Of the 22 recruited participants, 7 were eliminated with a CAIT score of 24 or higher, and a total of 15 participants participated. The revised Waterloo Footedness Questionnaire was used to select the dominant foot for forward and backward lunges to 15 participants who met the selection criteria [20]. In the starting position of the lunge movement, the lower limbs were spread to the pelvis width. The upper body was stretched, and both hands were put together. During the lunge movement, an eccentric contraction for two seconds and an concentric contraction for two seconds were adjusted to four beats of a metronome at $60 \mathrm{bpm}$ for four seconds [21]. Lunge stepping distance was standardized 
to $70 \%$ of dominant-leg length, that measured from the greater trochanter to the lateral malleolus. The non-predominant feet allowed the body to descend with the feeling of touching the ground. The dominant foot moving forward or backward was instructed to move in the horizontal plane. After sufficient training on the lunge movement before the measurement, the posture was practiced three to five times. A three-minute break was taken between each movementto minimize muscle fatigue due to repeated measurements. The ROM, muscle activity, and VGRF were measured during the forward and backward lunges, and the average value was measured three times in total and used for data analysis.

\section{Outcome measure}

\section{Range of motion}

A video was recorded using a smartphone (Galaxy S20, Samsung, Korea) to measure the ROM of the joint during lunge movement.A tripod was placed $2 \mathrm{~m}$ away from the participant, and the tripod height was $70 \mathrm{~cm}$ above the floor.Markers were attached to the greater trochanter, $3 \mathrm{~cm}$ lateral to the patella, and lateral malleolus. A video of the lunge movement performed by the researcher's verbal command "Lunge" was recorded using a smartphone. The recorded images were analyzed using the digital video analysis software program (Dartfish 10, Dartfish, Switzerland) to analyze the angles of the participant's hip, knee, and ankle during the lunge movements. The test-retest reliabilityof the Dartfish software program showed high reliability with $\mathrm{ICC}=0.79 \sim 0.91$ [22].

\section{Muscle activity}

An 8-channel surface electromyography-measuring device NoraxonMyosystem(1200 EMG, Noraxon Inc., USA) was used to measure the muscle activity of the VMO, VL, gluteus maximus (GM), and biceps femoris (BF) that act on posture maintenance during lunge movement. The EMG signals were collected by setting the sampling rate to $1,000 \mathrm{~Hz}$ and the frequency bandwidth to $40-250 \mathrm{~Hz}[23]$. The collected signal was subjected to a full-wave rectification, and the root mean square was stored in a computer. All data were processed using MyoReserach(2.02 software, Noraxon
Inc., USA). During the forward and backward lunges, the VMO electrode was attached $2 \mathrm{~cm}$ to the comfort side of the knee bone, and the VL electrode was attached 3 to $5 \mathrm{~cm}$ on the lateral side of the knee bone based on the midline of the femur [24]. The electrode of the GM was attached to the midline of the muscles located between the second sacral vertebrae and the great trochanter. The electrode of the BF was attached to the midline of the muscles located between the lateral epicondyle and the sciatic nodule of the femur [24]. maximal voluntary isometric contraction (MVIC) was measured and quantified to standardize the EMG signals of the four muscles used for measurement [25].

\section{Vertical Ground reaction force}

The VGRF, according to the forward and backward lunge movements, was measured using a Will balance board (WBB, Nintendo, Japan). The WBB is a force plate measuring $20.5 \times 13.2 \times 3.2$ inches. The change in weight and center of gravity can be observed using four load cells located at the corners. In addition, because of its low price and convenient portability, it is used to measure the movement of the VGRF and center of pressure $(\mathrm{CoP})$ in clinical practice. The WBB is equipment with very high validity $(\mathrm{ICC}=0.701 \sim$ $0.994)$ and reliability between inspection and retest (ICC $=0.676 \sim 0.946)$ compared to the existing widely used force plate [26]. During the forward and backward lunge movements, the VGRF was measured by placing the WBB on the dominant and non-dominant feet, respectively (Figure 1). The data collected through WBB was stored on a computer connected via Bluetooth using a balancia program (Balancia, Mintosys Inc., Republic of Korea). The average value measured three times in total was extracted with Excel and calculated by integrating with the VGRF value over time. The highest peak value was used for data analysis (Figure 2).

\section{Statistical analysis}

SPSS for Windows (ver 25.0, IBM Co., USA) was used for statistical analysis. The general characteristics of the participants are reported using the mean and standard deviation through descriptive statistics. The ROM, muscle activity, and VGRF between the forward 


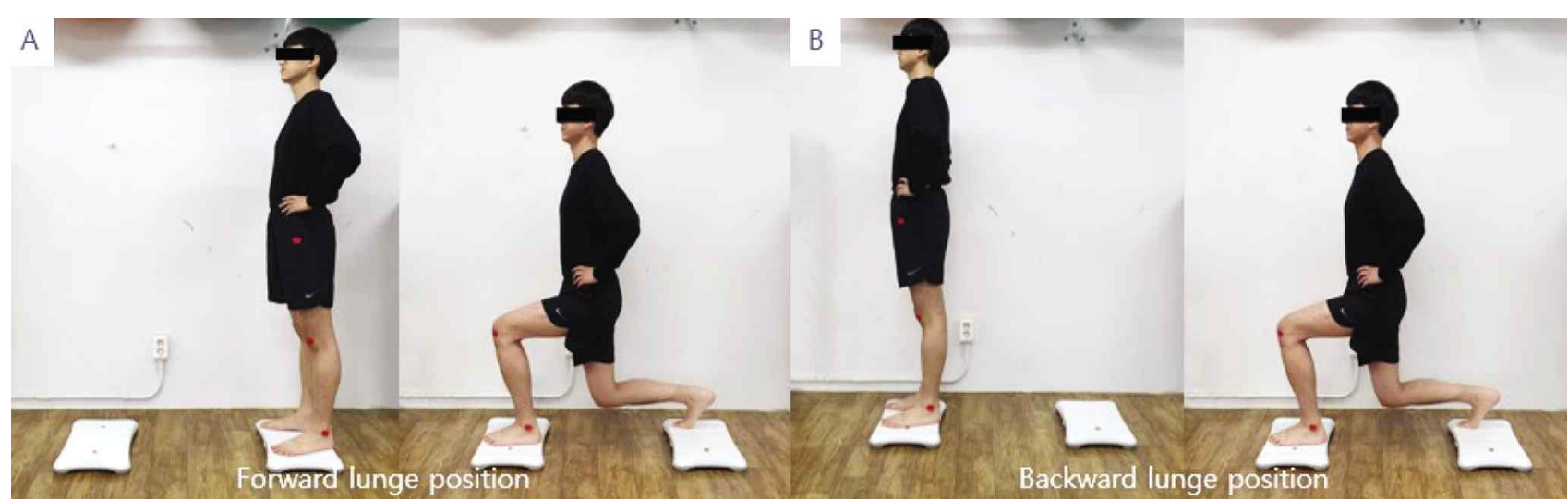

Figure 1. Forward lunge position (A) and Backward lunge position (B)

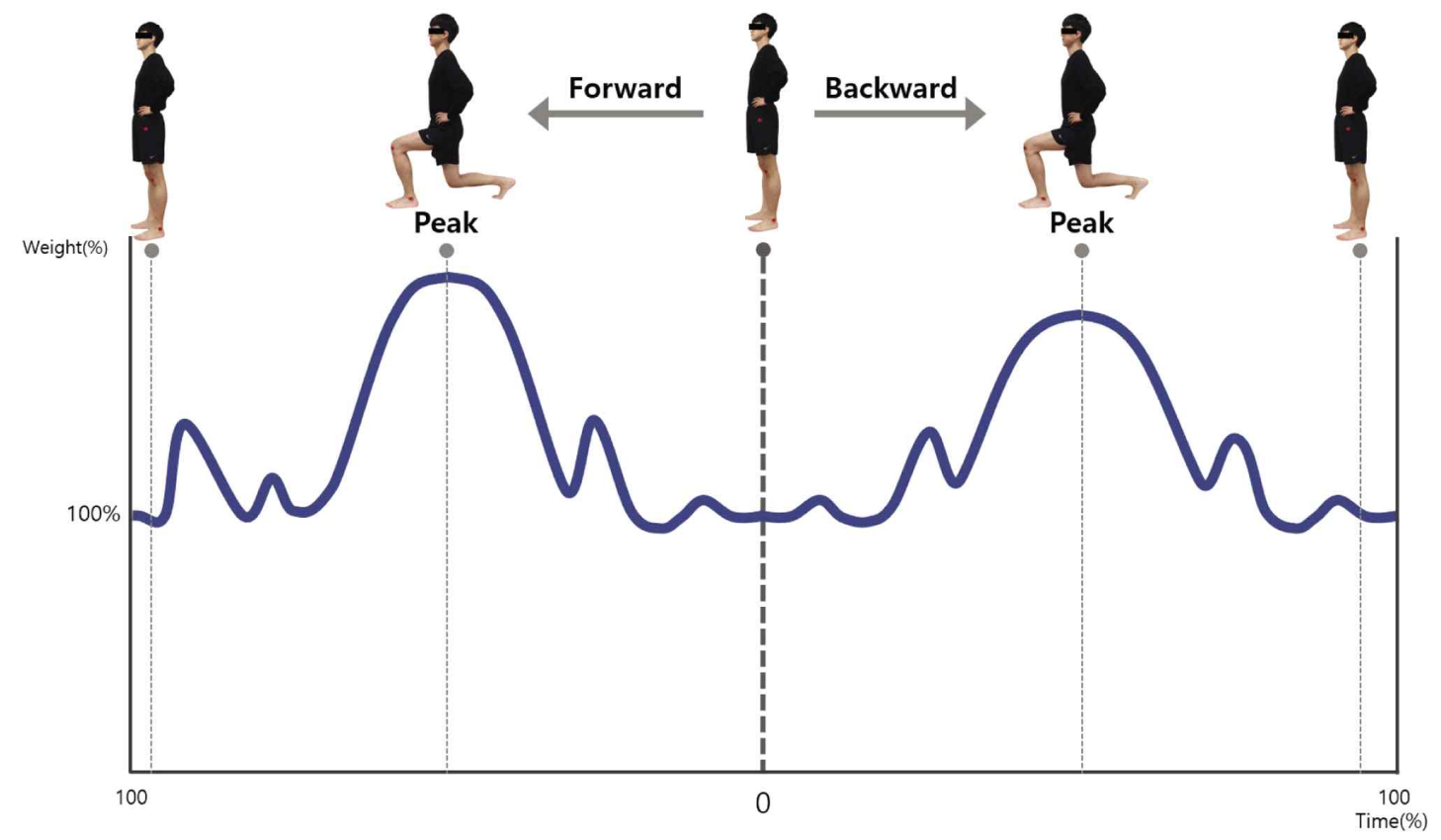

Figure 2. Vertical ground reaction force

lunge and the backward lunge were compared by comparative analysis using the paired t-test. Statistical significance was set at $\mathrm{p}<0.05$.

\section{Results}

Table 1 lists the general characteristics of the 15 participants in the study. A significant increase in the ROM of the knee and ankle was observed during the forward and backward lunges $(\mathrm{p}<0.05)$. In addition, in terms of the muscle activity, the peak values of the VMO and VGRF also showed a significant increase in the forward lunge compared to the backward lunge $(p<0.05)$ (Table 2).

\section{Discussion}

This study examined the kinematic relationship and difference through the ROM, muscle activity, and VGRF during forward and backward lunge movements in 15 adult males in their twenties. Significant differences in the knee, ankle ROM, muscle activity in VMO, and peak values of VGRF were confirmed in the forward lunge compared to the backward lunge $(p<0.05)$. Among the $\mathrm{CKC}$ exercises, squat and lunge are the most frequently performed full-body exercises. The lunge provides more left and right instability than the squat, providing considerable stimulation to various muscles of the lower limb [27]. The weight is placed on the 
Table 1. General characteristics of the participants

$(\mathrm{n}=15)$

\begin{tabular}{ll}
\hline General characteristic & Participant \\
\hline Age $($ Years $)$ & $29.47 \quad(3.81)$ \\
Height $(\mathrm{cm})$ & $173.93(5.78)$ \\
Weight $(\mathrm{kg})$ & $72.44 \quad(9.25)$ \\
BMI $\left(\mathrm{kg} / \mathrm{m}^{2}\right)$ & $23.94 \quad(2.80)$ \\
Dominantfoot $(\mathrm{Rt} / \mathrm{Lt})$ & $13 / 2$ \\
\hline
\end{tabular}

Values are presented as mean (SD).

BMI: Body mass index

Table 2. Comparison of the dependent variables according to the intervention between the groups.

\begin{tabular}{lllll}
\hline Dependent variables & Forward lunge & Backward lunge & Diff & t(p) \\
\hline Range of motion $\left(^{\circ}\right)$ & & & & $-2.042(0.060)$ \\
Hip & $98.74(12.86)$ & $93.83(11.90)$ & $-4.92(9.32)$ & $-2.267(0.040)^{*}$ \\
Knee & $105.49(5.18)$ & $99.52(8.22)$ & $-5.97(10.20)$ & $-2.211(0.044)^{*}$ \\
Ankle & $15.81(4.50)$ & $13.74(4.13)$ & $-2.07(3.62)$ & $-.850(0.410)$ \\
\hline Electromyography $(\%)$ & & & $-1.26(5.72)$ & $-1.121(0.281)$ \\
BF & $14.77(9.22)$ & $13.51(9.66)$ & $-5.81(20.07)$ & $-2.459(0.028)^{*}$ \\
VL & $36.96(22.61)$ & $31.15(20.21)$ & $-8.51(13.40)$ & $-1.941(0.073)$ \\
VMO & $54.28(23.71)$ & $45.77(16.18)$ & $-1.28(2.56)$ & $-2.340(0.035)^{*}$ \\
GM & $16.99(7.69)$ & $15.71(6.56)$ & & $-7.67(12.70)$ \\
\hline Ground reaction force $(\mathrm{W}, \%)$ & $142.81(13.26)$ & $135.14(13.80)$ & &
\end{tabular}

Values are presented as mean (SD).

BF: biceps femoris, VL: vastus lateralis, VM: vastus medialis oblique, GM: gluteus maximus.

$* p<0.05$

supporting lower limbs, absorbs the propulsion, and is stored as elastic energy, which is converted to returning propulsion [28]. Latash [29] reported that the front lower limb supports the ground with the sole during the lunge movement, while the rear lower limb supports the ground with the heel upright. Hence, it is effective for simultaneous contraction for joint stabilization because the exercise is performed in an unstable state.

In the case of a general standard lunge, hip flexion $90^{\circ}$, knee flexion $100^{\circ}$, and ankle dorsiflexion $12^{\circ}$ appear on average at the maximum bending angle [30]. In this study, the average knee flexion was $105.49^{\circ}$, and the ankle dorsiflexion was $15.81^{\circ}$ in the forward lunge, showing a significant difference compared to the backward lunge $(p<0.05)$. Although there was no significant difference in hip flexion, there was a significant difference with an average hip flexion of $98.74^{\circ}$ at the forward lunge and $93.93^{\circ}$ in the backward lunge. Goulette et al. [13] reported a significant difference in the average knee-bending angle and the maximum knee-bending angle during a forward lunge according to the lunge direction, which is consistent with the present results.In this study, the primary role of the dominant foot is the dynamic motion of stepping on the foot when performing a forward lunge. The main role is the static role supported by the dominant foot when performing the backward lunge. Therefore, the difference in the angle according to the lunge motion is caused by the difference in the process for performing the motion of the lower limb.

The lunge, a typical CKC exercise, can increase the muscle activity of VMO and VL relatively significantly 
because it uses force more intensively on only one lower extremity than a squat [31]. According to the study ofComfort et al. [32], the compressive load and shear force of the tibiofemoral joint in the forward lunge increased gradually as the knee flexion angle increased compared to the backward lunge.In a study by $\mathrm{Kim}$ et al. [33], the muscle activity of VMO and $\mathrm{VL}$ was higher than that of the rectus femoris during the forward lunge $(p<0.05)$. In addition, according to the position of the ankle, the muscle activity of the VMO had the largest activity among the thighs.In this study, the forward lunge showed a significant difference in the VMO compared to the backward lunge $(p<0.05)$. The dominant foot in the front plays a static role during the backward lunge, while during the forward lunge, the dominant foot plays a dynamic role that extends forward.It is thought that the activity of VMO increased due to the momentary momentum.

During CKC exercise, VGRF can explain the exercise load during lunge movement by the action and reaction with the ground. Wurm et al. [34] argued that the efficiency increased with increasing ground repulsion when performing exercises to increase muscle strength. According to Comfort et al. [35], there was no significant difference in the maximum eccentric contraction and the efferent contraction in the forward and backward lunges. They recommended the posterior lunge rather than the forward lunge for PFPS patients. Goulette et al. [13] reported that the joint load on the knee joint during the forward lunge was significantly greater than that of the backward lunge. In this study, there was a significant difference in the peakvalue of VGRF in the forward lunge compared to the backward lunge $(p<0.05)$. Therefore, the VGRF should be considered according to the situation of the participant, consistent with the direction of movement during lunge movement in relation to the load on the knee joint.

Based on these results, compared to the backward lunge, the forward lunge hasclinical utility as an effective method to improve the ROM of the knee and ankle, muscle activity of VMO, and peak value of VGRF. This study had several limitations. First, the study participants were limited to adult males in their twenties due to errors in motion analysis during lunge movement. Therefore, it was somewhat difficult to generalize men and women of all ages and the elderly.
Second, there was no limit on the interval according to the length of the lower limbs during the forward and backward lunge. Third, there was no investigation of the posterior lower extremities during the lunge. Therefore, continuous research will be needed to supplement these limitations and suggest a more efficient lunge exercise method.

\section{Conflicts of interest}

No potential conflict of interest relevant to this article was reported.

\section{References}

1. Guerra ML, Singh PJ, Taylor NF. Early mobilization of patients who have had a hip or knee joint replacement reduces length of stay in hospital: a systematic review. ClinRehabil. 2015;29:844-54.

2. Gustafson JA, Anderton W, Sowa GA, Piva SR, Farrokhi S. Dynamic knee joint stiffness and contralateral knee joint loading during prolonged walking in patients with unilateral knee osteoarthritis. GaitPosture. 2019;68:44-9.

3. Chandler TJ, Brown LE. Conditioning for strength and human performance. Philadelphia: Wolters Kluwer Health; 2008.

4. Lam $\mathrm{S}, \mathrm{Ng}$ T. First simultaneous isolation of a ribosome inactivating protein and an antifungal protein from a mushroom (Lyophyllum shimeji) together with evidence for synergism of their antifungal effects. Arch BiochemBiophys. 2001;393:271-80.

5. Witvrouw E, Danneels L, Asselman P, D'Have T, Cambier D. Muscle flexibility as a risk factor for developing muscle injuries in male professional soccer players: a prospective study. Am JSports Med. 2003;31:41-6.

6. Pandey RS, Yadav T, Rayjade A, Chintamani R, Kolhatkar A. Effectiveness of Open Kinematic Chain Exercises and Closed Kinematic Chain Exercises in Adductor Groin Pain in Recreational Football Players. J Indian Forensic Sci. 2020;14:276-82.

7. Ha TW, Park SH, Lee MM. Comparison of difference in muscle activity ratio, ground reaction force and knee valgus angle during single leg squat and landing according to dynamic taping. Phys TherRehabil 
Sci. 2020;9:281-6.

8. Park SH, Lee MM. Effects of progressive neuromuscular stabilization exercise on the support surface on patients with high obesity with lumbar instability: A double-blinded randomized controlled trial. Medicine (Baltimore). 2021;100:e23285.

9. Park SH, Lee MM. Effects of a Progressive Stabilization Exercise Program Using Respiratory Resistance for Patients with Lumbar Instability: A Randomized Controlled Trial. Med Sci Monit. 2019;25:1740-8.

10. Harato K, Sakurai A, Morishige Y, Kobayashi S, Niki Y, Nagura T. Biomechanical correlation at the knee joint between static lunge and single-leg drop landing-a comparative study among three different toe directions. J Exp Orthop. 2019;6:1-7.

11. Doma K, Leicht AS, Boullosa D, Woods CT. Lunge exercises with blood-flow restriction induces post-activation potentiation and improves vertical jump performance. Eur J Appl Physiol. 2020;120:687-95.

12. Nayanti AP, Prabowo T, Sari DM. The Effects of Kinesio Taping and Quadriceps Muscle Strengthening Exercise on Quadriceps Muscle Strength and Functional Status in Knee Osteoarthritis. JMed Health. 2020;2.

13. Goulette D, Griffith P, Schiller M, Rutherford D, Kernozek TW. Patellofemoral joint loading during the forward and backward lunge. Phys TherSport. 2021;47:178-84.

14. De Roeck J, Van Houcke J, Almeida D, Galibarov P, De Roeck L, Audenaert EA. Statistical modeling of lower limb kinetics during deep squat and forward lunge. Front BioengBiotechnol. 2020;8:233.

15. Paladino A, Marchetti F, Ponzoni L, Colombo G. The Interplay between Structural Stability and Plasticity Determines Mutation Profiles and Chaperone Dependence in Protein Kinases. J Chem Theory Comput. 2018;14:1059-70.

16. Marchetti PH, Guiselini MA, da Silva JJ, Tucker R, Behm DG, Brown LE. Balance and lower limb muscle activation between in-line and traditional lunge exercises. J Hum Kinet. 2018;62:15-22.

17. Viveiro LAP, Gomes GCV, Bacha JMR, Junior NC, Kallas ME, Reis M, et al. Reliability, validity, and ability to identity fall status of the Berg Balance Scale, Balance Evaluation Systems Test (BESTest),
Mini-BESTest, and Brief-BESTest in older adults who live in nursing homes. J Geriatr Phys Ther. 2019;42:45-54.

18. Ko J, Rosen AB, Brown CN. Cross-cultural adaptation, reliability, and validation of the Korean version of the identification functional ankle instability (IdFAI). DisabilRehabil. 2018;40:3185-90.

19. Wu HW, Tsai CF, Liang KH, Chang YW. Effect of loading devices on muscle activation in squat and lunge. J Sport Rehabil. 2020;29:200-5.

20. Yang N, Waddington G, Adams R, Han J. Translation, cultural adaption, and test-retest reliability of Chinese versions of the Edinburgh Handedness Inventory and Waterloo Footedness Questionnaire. Laterality: Asymmetries of Body, BrainCogn. 2018;23:255-73.

21. Dill KE, Begalle RL, Frank BS, Zinder SM, Padua DA. Altered knee and ankle kinematics during squatting in those with limited weight-bearing-lunge ankle-dorsiflexion range of motion. J Athl Train. 2014;49:723-32.

22. Alahmari A, Herrington L, Jones R. Concurrent validity of two-dimensional video analysis of lower-extremity frontal plane of movement during multidirectional single-leg landing. Phys TherSport. 2020;42:40-5.

23. Park HY, Kim NH, Cha YJ. Comparison of Muscle Activity in Proximal Muscle of Lower Extremities during Lunge according to the Anterior-posterior Distance of Foot Position. J Korean Soc Phys Med. 2018;13:131-8.

24. Cram JR. The history of surface electromyography. Appl Psychophysiol Biofeedback. 2003;28:81-91.

25. Alenabi T, Whittaker R, Kim SY, Dickerson CR. Maximal voluntary isometric contraction tests for normalizing electromyographic data from different regions of supraspinatus and infraspinatus muscles: Identifying reliable combinations. J ElectromyogrKinesiol. 2018;41:19-26.

26. Yang SR, Oh YR, Jeon YR, Park DS. Test-retest reliability of sit-to-stand and gait assessment using the wii balance board. Phys Ther Korea. 2016;23:40-7.

27. Boudreau SN, Dwyer MK, Mattacola CG, Lattermann C, Uhl TL, McKeon JM. Hip-muscle activation during the lunge, single-leg squat, and step-up-and-over exercises. J Sport Rehabil. 2009;18:91-103. 
28. Cronin J, McNAIR P, MARSHALL R. Lunge performance and its determinants. J Sports Sci. 2003;21:49-57.

29. Latash ML. Muscle coactivation: definitions, mechanisms, and functions. JNeurophysiol. 2018;120:88-104.

30. Riemann B, Congleton A, Ward R, Davies G. Biomechanical comparison of forward and lateral lunges at varying step lengths. J Sports Med Phys Fitness. 2013;53:130-8.

31. Ekstrom RA, Donatelli RA, Carp KC. Electromyographic analysis of core trunk, hip, and thigh muscles during 9 rehabilitation exercises. J Orthop Sports Phys Ther. 2007;37:754-62.

32. Comfort P, Jones PA, McMahon JJ, Newton R. Effect of knee and trunk angle on kinetic variables during the isometric midthigh pull: Test-retest reliability. Int J Sports Physiol Perform. 2015;10:58-63.

33. Kim JH, Park SK, Kang JI, Yang DJ. Effects of lumbar stability exercise program on trunk, lower extremity of muscle activity and balance in soccer player. JKorean PhysTher. 2010;22:25-31.

34. Wurm B, Garceau L, Zanden T, Fauth M, Ebben W. Ground reaction force and rate of force development during lower body resistance training exercises. ISBS-Conference Proceedings Archive; 2010 Jul 19-23; Michigan, USA.

35. Comfort P, Jones PA, Smith LC, Herrington L. Joint kinetics and kinematics during common lower limb rehabilitation exercises. JAthlTrain. 2015;50:1011-8. 